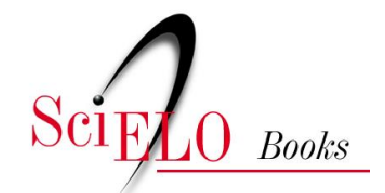

\title{
Capítulo 12 - Apuntes para una ecología política de la integración en América Latina y el Caribe
}

\author{
José Esteban Castro
}

\section{SciELO Books / SciELO Livros / SciELO Libros}

CASTRO, J. E. Apuntes para una ecología política de la integración en América Latina y el Caribe. In: Água e democracia na América Latina [online]. Campina Grande: EDUEPB, 2016, pp. 397-427. ISBN 978-85-7879-486-6. Available from: doi: 10.7476/9788578794866.0013. Also available in ePUB from: http://books.scielo.org/id/tn4y9/epub/castro-9788578794866.epub.

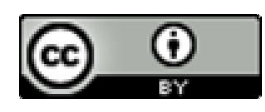

All the contents of this work, except where otherwise noted, is licensed under a Creative Commons Attribution $\underline{4.0 \text { International license. }}$

Todo o conteúdo deste trabalho, exceto quando houver ressalva, é publicado sob a licença Creative Commons Atribição 4.0.

Todo el contenido de esta obra, excepto donde se indique lo contrario, está bajo licencia de la licencia Creative Commons Reconocimento 4.0. 


\section{Apuntes para una ecología política de la integración en América Latina y el Caribe ${ }^{1}$}

\section{Introducción}

América Latina y el Caribe pasan indudablemente por un momento excepcional en su historia en relación a las perspectivas de mayor integración de sus pueblos a través de la profundización de las relaciones económico-financieras, comerciales, culturales, de defensa, de infraestructura y, crucialmente, políticas. Es importante reconocer el carácter apasionante de la experiencia actual de los países de la región, especialmente, aunque no solamente en América del Sur. No es necesario excedernos en el romanticismo ni tampoco ignorar las contradicciones, errores, obstáculos y peligros, para partir de un hecho difícil de negar objetivamente: la región enfrenta el Siglo XXI en condiciones inéditamente favorables a la consolidación de procesos de integración con grados de autonomía potencialmente importantes. En el plano institucional no puedo dejar de mencionar como ejemplos los procesos de integración en América del Sur, con la consolidación del funcionamiento de algunas esferas de acción del Mercado Común del Sur (MERCOSUR), incluyendo sus subregiones, y la creación de la Unión de Naciones Suramericanas (UNASUR) y a nivel del continente el lanzamiento de la Comunidad de Estados Latinoamericanos y del Caribe (CELAC). Indudablemente incluso la persona más escéptica o crítica de estos procesos deberá admitir que los mismos representan avances incuestionables en

1 Una versión previa fue publicada en el Anuario de la Red de Investigadores sobre América Latina y el Caribe 2010, Guadalajara, Mexico: University of Guadalajara (UdG), 2014, pp.479-502. Otras versiones fueron publicadas en Bolivia, España y México. 
varias dimensiones del proceso de integración, notablemente en la coordinación de acciones entre países a nivel político institucional.

Ahora bien, en este breve trabajo me preocupa discutir fundamentalmente algunas cuestiones relativas a la relación entre los procesos de integración y de democratización substantiva de las sociedades de la región, colocando el énfasis en los conflictos derivados de las desigualdades e injusticias socio-ambientales, percibidas o demostradas, que emergen en la mayoría de los países en el marco de dichos procesos. Mi argumento central en este capítulo consiste en afirmar que uno de los obstáculos y amenazas más complejos que enfrenta la integración democrática de la región, y en realidad el futuro mismo de la democracia substantiva en nuestros países, es la destrucción de la base material de nuestras sociedades con la consecuente profundización de conflictos vinculados con la injusticia y la desigualdad socio-ambientales. Quiero aclarar que mi posicionamiento no parte del ambientalismo ni del ecologismo, sino que como sugiere el título del capítulo está anclado en los presupuestos de la ecología política. En esta perspectiva, la incorporación de la dimensión ambiental en el análisis y en la acción constituye un elemento fundamental en la lucha por la emancipación humana, particularmente la lucha contra la desigualdad y la injusticia en relación al acceso a los bienes y servicios y la protección contra las amenazas y peligros conectados con los procesos socio-naturales. El proceso de integración latinoamericano y caribeño, entendido como un proceso democrático en sentido substantivo y no meramente formal, confronta en la problemática socio-ambiental uno de sus obstáculos más importantes y difíciles de superar.

\section{Con el objeto de facilitar el análisis he ordenado el texto en la forma de cuatro proposiciones.}

Proposición No 1. Con independencia de la heterogeneidad de las tradiciones intelectuales y políticas y de las condiciones específicas que caracterizan a los distintos países y regiones, la dinámica central del proceso de integración yace en el avance y profundización de relaciones 
capitalistas de producción, distribución y consumo, y sus correspondientes expresiones socio-culturales y politicas. La evidencia sugiere que el proceso de integración está dinamizado fundamentalmente por la profundización de relaciones capitalistas, tanto sea mediante la constitución de algunas autonomías emergentes como por la consolidación y expansión de heteronomías prexistentes.

Proposición No 2. La consolidación y avance de relaciones capitalistas ha estado históricamente vinculada con la destrucción y la expoliación, con efectos muchas veces irreversibles, de la base material de las sociedades humanas. El pasado de América Latina y el Caribe ha sido marcado por el intercambio ecológico desigual con las grandes potencias imperialistas, que está en la base de la deuda ecológica acumulada desde el siglo dieciséis por dichos países y que incluye formas de depredación menos difundidas como el imperialismo tóxico y la biopiratería.

Proposición No 3. El proceso de integración en Sudamérica, con efectos potenciales en el resto de América Latina y el Caribe, está fundado en la consolidación y rápida expansión del crecimiento económico motorizado por un rol activo del estado en apoyar a los actores capitalistas nacionales y transnacionales en los países que lideran el proceso. La creciente autonomía de los estados de la región, reforzada en gran medida por el proceso de integración política, tiende a re-equilibrar en cierta medida la situación histórica de intercambio económico desigual que había sido profundizada por las políticas neoliberales desde la década de 1980. Sin embargo, las formas que asume el proceso de integración en el plano económico-productivo amenazan con profundizar las relaciones de intercambio ecológico desigual entre países y regiones y al interior de los propios países.

Proposición No 4. La destrucción de la base material que acompaña el proceso de intercambio ecológico desigual se presenta como una doble amenaza para el proceso de integración democrática en la región. Por una parte, la destrucción de la base material por definición constituye un 
obstáculo al propio modelo de integración fundado en la profundización de relaciones capitalistas, ya que erosiona los mismos fundamentos de la producción. Por otra parte, el proceso se ha constituido en uno de los motores de la producción y reproducción de desigualdades estructurales, en la forma de injusticia y desigualdad socio-ambientales, y como tal, en un obstáculo crucial al proceso de profundización de la democracia substantiva.

\section{Integración capitalista}

Proposición No 1. Con independencia de la heterogeneidad de las tradiciones intelectuales y políticas y de las condiciones específicas que caracterizan a los distintos países y regiones, la dinámica central del proceso de integración yace en el avance y profundización de relaciones capitalistas de producción, distribución y consumo, y sus correspondientes expresiones socio-culturales y políticas. La evidencia sugiere que el proceso de integración está dinamizado fundamentalmente por la profundización de relaciones capitalistas, tanto sea mediante la constitución de algunas autonomías emergentes como por la consolidación y expansión de heteronomías prexistentes.

El análisis del proceso de integración nos invita a plantear una serie de cuestiones básicas: ¿qué integración? ¿Integración de qué y de quiénes? ¿Integración para qué y para quiénes? ¿Integración a qué costo? El costo, ¿es un costo para quién? No pretendo dar respuesta plena a esas preguntas en un breve capítulo, pero las mismas apuntan a una serie de cuestiones centrales que no pueden ser soslayadas aun cuando se defienda una visión positiva, incluso optimista del proceso de integración. Al menos es importante plantearse estas y otras cuestiones similares en el marco del necesario debate racional que debe acompañar al proceso. En ese sentido, por ejemplo, a pesar de la retórica emitida desde el grupo de países que componen la Alianza Bolivariana para las Américas (ALBA), la dinámica de la integración regional es motorizada por el proceso de acumulación capitalista global, que impone determinaciones incluso a los países de dicha alianza, que defienden 
la construcción de un "socialismo del siglo XXI". Esto no implica negar los avances de la democracia directa en Venezuela ni desde luego los logros históricos de Cuba en una serie de áreas clave para la democratización substantiva de la sociedad como son la educación o la salud. Igualmente, no se trata de ignorar el avance significativo logrado por varios otros países de la región en la rápida reversión de las condiciones extremas de pobreza y la mejora del ingreso para amplios sectores de la población, hechos suficientemente documentados (CEPAL 2009; Cornia 2010; López-Calva y Lustig 2010). Sin embargo, es muy importante despejar equívocos y desmitificar discursos: los procesos de integración en marcha en América Latina y el Caribe están insertos en y son sobre determinados por las dinámicas del proceso de acumulación capitalista de escala global, que se encuentra en un momento de acelerada expansión y profundización en la región. Al mismo tiempo que deben reconocerse los avances democráticos concretos, logrados en un marco de gran heterogeneidad de formas culturales, institucionales, políticas y socio-económicas que caracterizan el proceso de integración en cada territorio nacional y regional, debe también señalarse que el carácter capitalista del proceso entraña consecuencias a distintos niveles, ciertamente en el plano socio-ambiental, que no pueden ignorarse. Vale la pena recordar aquí una conclusión de Norbert Elias sobre la tensión permanente entre la práctica y la estrategia, el corto y el largo plazo, y sobre todo en la relación entre conocimiento y acción en los procesos políticos:

"los gobiernos contemporáneos suelen afirmar -quizás en buena fe- que pueden superar los agudos problemas sociales de sus países 'racionalmente' o 'realísticamente'. Sin embargo, en la práctica lo que suelen hacer es llenar las lagunas de nuestro conocimiento empírico rudimentario sobre las dinámicas de las interrelaciones sociales con doctrinas dogmáticas, panaceas dictadas, o intereses partidarios de corto plazo. Tomando decisiones mayormente al azar, permanecen a la merced de los eventos, cuya secuencia los gobiernos entienden tan poco como su gobernados" (Elias 1978: 31). 
Sintonizando la reflexión de Elías con el proceso de integración que analizamos, no se trata de cuestionar el compromiso político o ideológico ni las intenciones de líderes políticos, gobiernos o alianzas de países, para mencionar solamente a algunos de los actores relevantes, sino de la necesidad de un análisis objetivo de los procesos estructurales que enmarcan el proceso de integración al margen de las voluntades y deseos humanos. En la perspectiva de este artículo, esto implica muy particularmente la necesidad de mayor conocimiento acerca de las interrelaciones entre el proceso de integración democrática y la dimensión socio-ambiental.

En relación con esto, la segunda pregunta, ¿integración de qué y de quiénes?, apunta precisamente a destacar la elevada heterogeneidad de regiones y actores, de tradiciones culturales y políticas, escalas, condiciones físico-naturales, procesos de formación estatal e institucional, y configuraciones de poder intra-, inter-, y trans estatal, entre otras cuestiones. Muy particularmente, entraña una consideración de las diversas tradiciones y prácticas históricas, incluyendo valores y expresiones culturales, de las relaciones que los distintos grupos humanos han establecido con la base material de sus sociedades en las regiones de América latina y el Caribe. Por ejemplo, en el proceso de integración tenemos a los países y regiones andinos, en algunos de los cuales se ha dado un proceso radical de democratización que al menos en el plano del discurso da centralidad a tradiciones y valores culturales (como los del "Buen Vivir" en Bolivia y Ecuador, ver por ejemplo: Farah y Vasapollo 2011; Gudynas 2011) que no tienen un correlato fuerte en las tradiciones prevalecientes en sus vecinos del Cono Sur. Al mismo tiempo, dichas tradiciones andinas también contrastan fuertemente con las dominantes en, solo por mencionar algunos casos, los diversos territorios del Brasil o de las áreas de México, Centroamérica y el Caribe. No se trata aquí de celebrar unas tradiciones sobre otras o establecer jerarquías entre ellas, sino simplemente de reconocer la extremadamente compleja diversidad de lo que se intenta integrar incluso si reducimos el análisis a la dimensión socio-ambiental del proceso.

Desde otro ángulo, en relación a la configuración del poder relativo de los distintos países en las relaciones inter y transnacionales, la integración 
tiene lugar en el marco de la constitución de autonomías capitalistas emergentes y en la consolidación simultánea de heteronomías prexistentes, procesos que se encuentran interconectados. La emergencia de Brasil como un poder económico global y su consolidación como "locomotora" del crecimiento en América del Sur es sin duda el ejemplo más destacado de una autonomía capitalista emergente en pleno proceso de constitución. Por otra parte, la consolidación de heteronomías prexistentes se da notoriamente en el caso de México y la región centroamericana, que desde la década de 1990 ha visto crecer su dependencia con los Estados Unidos, ya sea a través del Tratado de Libre Comercio entre México, Estados Unidos y Canadá iniciado en 1994 como también mediante otra serie de procesos que incluyen la dependencia de países enteros de las remesas enviadas por sus emigrantes y la militarización regional bajo control de los Estados Unidos en el marco de la "guerra contra las drogas", tomando en cuenta que esta última desde luego abarca también a países de América del Sur y particularmente Colombia (Delgado Wise y Ortega Breña 2006; Gammage 2006; Suárez Salazar y Ortega Breña 2007).

Por último, más allá de la retórica de la "integración de los pueblos", que compartimos como ideal y como proyecto político, el análisis desapasionado nos sugiere que en las dimensiones centrales del proceso de integración en marcha prevalece la integración de intereses particulares, no siempre representantes de las mayorías populares, y no en menor medida los intereses de agentes sociales externos a la propia región que se integra. Estos son fundamentalmente intereses capitalistas, es decir, intereses que privilegian la acumulación privada de la ganancia y no la distribución social de los beneficios del desarrollo. Retomaremos este punto con algunos ejemplos posteriormente. Si se acepta la primera proposición sobre el carácter esencialmente capitalista del proceso de integración, podemos examinar en mayor detalle los obstáculos y amenazas que enfrenta el proceso de integración, entendido como un proceso democrático en sentido substantivo, no meramente formal. 


\section{Las condiciones históricas}

Proposición $\mathrm{N}^{\circ}$ 2. La consolidación y avance de relaciones capitalistas ha estado históricamente vinculada con la destrucción y la expoliación, con efectos muchas veces irreversibles, de la base material de las sociedades humanas. El pasado de América Latina y el Caribe ha sido marcado por el intercambio ecológico desigual con las grandes potencias imperialistas, que está en la base de la deuda ecológica acumulada desde el siglo dieciséis por dichos países y que incluye formas de depredación menos difundidas como el imperialismo tóxico y la biopiratería.

Aunque sabemos que los seres humanos siempre han transformado el medio natural, con frecuencia con resultados negativos para el medio y para las propias sociedades humanas (De Vries y Goudsblom 2002), la relación entre la expansión global del sistema capitalista y el incremento cuantitativo y cualitativo de los procesos de destrucción de la base material de la vida, cuando no de la vida misma, está suficientemente documentado. Particularmente el Siglo XX constituyó una etapa de grandes transformaciones antropogénicas del medio ambiente con consecuencias para la vida en general y para la especie humana en particular que aún no alcanzamos a entender ni explicar cabalmente (McNeill 2000; Fischer-Kowalski y Haberl 2007). Desde luego, la relación entre la producción capitalista y la destrucción ambiental, particularmente en relación a sus consecuencias para la vida y las posibles formas de resolución de los problemas (ej. reducción de la biodiversidad, contaminación, calentamiento global, agotamiento de bienes no renovables, etc.), han sido y siguen siendo objeto de un intenso debate. Aunque dado lo breve de este trabajo no podemos desarrollar este punto en detalle, cabe al menos mencionar por su relevancia para nuestro tema que existe un amplio abanico de posiciones y argumentos, respaldados en tradiciones intelectuales y políticas de larga trayectoria, sobre dichas cuestiones.

Para simplificar, tenemos en un extremo a los representantes del determinismo tecnológico a ultranza, para quienes la destrucción de la base 
material por parte del sistema capitalista tiene solución dentro del marco del propio sistema: la solución es más desarrollo tecnológico. En esta posición coinciden tanto defensores del capitalismo así como también algunos de sus críticos más acérrimos, hecho sofisticadamente analizado por el sociólogo portugués Hermínio Martins (Martins 1996; Martins 1998; Martins 1998; Martins 1998). En este extremo del debate, no importa si los árboles desaparecen o si el agua dulce se torna inservible para la vida, pues la tecnología nos permitirá crear soluciones artificiales, en última instancia incluso árboles y agua sintéticos, para resolver esas molestias. En realidad el propio cuerpo humano ya es objeto de dicha transformación tecnológica, con la capacidad creciente de remplazar tejidos e incluso órganos humanos por sustitutos artificiales. En la perspectiva de esta tradición de pensamiento es cuestión de tiempo y de políticas adecuadas que dejen en libertad completa a los actores capitalistas para que puedan desarrollar su inventiva y recrear el universo. Como lo dice el Director de Estudios de los Recursos Naturales del ultra liberal Instituto CATO en Estados Unidos: "No solamente el mundo es hoy sustentable, sino que es más sustentable que nunca antes, en el sentido de que las generaciones futuras serán herederas de un volumen mayor de capital natural y artificial que les permitirá satisfacer sus necesidades mucho mejor que las generaciones precedentes" (Taylor 1994: 49). Para Taylor, el debate de la sustentabilidad es una solución en busca de un problema. En el otro extremo, varias tradiciones intelectuales y políticas han insistido al menos desde el siglo diecinueve en el proceso inherentemente destructor de la base material que caracteriza al capitalismo, hecho que fue conceptualizado por James O'Connor como la "segunda contradicción del capitalismo" (ver también los trabajos de Schnaiberg y colegas: Schnaiberg 1980; Schnaiberg and Gould 1994; O'Connor 1998). Si la primera contradicción enfrenta al capital con el proletariado, en la segunda contradicción el capital se enfrenta con la destrucción de su propia base material de la que depende su existencia.

Cabe aclarar que la toma de conciencia sobre este proceso de destrucción de la base material que caracteriza al sistema capitalista no ha sido, como algunos parecen pensar, un tema de socialistas y ambientalistas 
románticos que no entienden o no quieren entender las realidades económicas de la vida social. Desde la profundización del debate internacional sobre la existencia de "límites" ecológicos a la producción capitalista que se dio desde fines de la década de 1960, empresarios e intelectuales comprometidos con la democracia capitalista también han estado activamente involucrados en la tarea de investigar y demostrar no solamente la existencia de tales límites sino incluso que esos límites ya han sido superados y que la especie humana estaría viviendo "a crédito", en términos ambientales. Desde luego, uno de los casos más notables y mejor conocidos es el del Club de Roma, que en 1972 publicó el ya clásico informe de investigación sobre "Límites al Crecimiento", un informe que cumple 40 años en 2012 y está dando lugar a un debate renovado (Meadows, Meadows et al. 1972; Club de Roma 2012).

Ahora bien, históricamente América Latina y el Caribe formaron parte de los territorios de la acumulación originaria ampliada de los países capitalistas centrales, y estuvieron sujetos a relaciones de intercambio desigual en los diversos planos, incluso el ecológico. Este hecho ha pasado del plano de la mera retórica antimperialista y anticolonialista para formar parte de estudios rigurosos que inclusive han producido estimaciones de la deuda ecológica que los países industrializados han adquirido con el Sur Global, no solamente a través del saqueo colonial directo sino también a través de las formas más sutiles del intercambio ecológico desigual (ver entre otros autores: Bunker 1984; Guha y Martínez-Alier 1997; Hornborg 1998; Martinez-Alier 2002; Martínez-Alier y Olivares 2003; Rice 2007; Rice 2007; Roberts y Parks 2007; Nelson y Robertson 2008; Jorgenson y Clark 2009; Rice 2009; Simms 2009).

Más recientemente, otros mecanismos de injusticia y desigualdad socio-ambiental, algunos también de larga data, han adquirido mayor peso como resultado de los avances tecnológicos en el marco del proceso de globalización capitalista, como por ejemplo el imperialismo tóxico y la biopiratería. El imperialismo tóxico se refiere a la transferencia, impuesta o negociada, de residuos tóxicos originados en un país, normalmente un país industrializado, a países pobres cuyos gobiernos aceptan el trato a cambio 
de una compensación o simplemente no tienen las condiciones de control y regulación que les permitan evitar la descarga de substancias tóxicas en sus territorios. En julio de 2009 el Diario Clarín publicó una noticia que ejemplifica el problema: "Descubrieron en tres aduanas brasileñas 1.600 toneladas con bolsas de sangre, preservativos, pañales usados y jeringas. Llegaban desde Gran Bretaña como plástico. El gobierno dice estar 'furioso' y ordenó repatriar los desechos" (Gosman 2009). Este es un pequeño ejemplo de una práctica bien documentada y extendida a nivel mundial (Martinez-Alier 2002). Sin embargo, el imperialismo tóxico también asume formas más sutiles, como por ejemplo la transferencia a otros países de cargas contaminantes integradas en los productos mercantilizados (Muradian, O'Connor et al. 2002).

Por su parte, la biopiratería, que consiste en la apropiación de material y conocimiento biológico sin reconocimiento ni compensación, es una práctica antigua constituyente del "imperialismo ecológico", un proceso en el cual los países de América Latina y el Caribe fungieron como un escenario central (Crosby 1988; sobre el caso específico de Argentina, ver por ejemplo: Brailovsky y Foguelman 2007). En tiempos recientes, la práctica ha alcanzado niveles elevados de sofisticación a través de los avances de la biotecnología y la ingeniería genética, notablemente en el campo de la bioprospección relacionada por ejemplo con la producción industrial de drogas comerciales y semillas genéticamente modificadas (Shiva 2001). En este sentido, un artículo publicado en enero de 2007 en los Estados Unidos sobre el tema de la bioprospección, discutía “¿quién tiene el derecho a las curas naturales?" (Vecchio 2007). El artículo se refería al hecho que una empresa privada había logrado obtener una patente exclusiva para comercializar productos basados en las propiedades medicinales de la maca, un tubérculo que crece en las alturas andinas y que las comunidades quechuas han utilizado por siglos para aliviar el mal de altura y como afrodisíaco. El de la maca también es un ejemplo pequeño de un proceso global, en el cual los países de América Latina y el Caribe se han convertido en un territorio privilegiado en las disputas en torno a la bioprospección/biopiratería, en gran medida debido a la rica biodiversidad que caracteriza a la región (Peritore y Niles 1992; Falcon y Fowler 2002; Helfrich 2002; Brailovsky y Foguelman 2007). 


\section{Integración e intercambio ecológico desigual}

Proposición $\mathrm{N}^{\circ}$ 3. El proceso de integración en Sudamérica, con efectos potenciales en el resto de América Latina y el Caribe, está fundado en la consolidación y rápida expansión del crecimiento económico motorizado por un rol activo del estado en apoyar a los actores capitalistas nacionales y transnacionales en los países que lideran el proceso. La creciente autonomía de los estados de la región, reforzada en gran medida por el proceso de integración política, tiende a re-equilibrar en cierta medida la situación histórica de intercambio económico desigual que había sido profundizada por las políticas neoliberales desde la década de 1980. Sin embargo, las formas que asume el proceso de integración en el plano económico-productivo amenazan con profundizar las relaciones de intercambio ecológico desigual entre países y regiones y al interior de los propios países.

El desarrollo económico está innegablemente relacionado con la creciente autonomía de los países de la región, particularmente los sudamericanos, y con el proceso de integración en marcha. Los aspectos positivos del proceso ya han sido comentados previamente y son objeto de un amplio reconocimiento internacional, particularmente en el caso de Brasil (OCDE 2010). Ahora bien, hay tres aspectos interconectados que deseo destacar en relación a esta tercera proposición y que tienen que ver con la continuidad, y en algunos aspectos profundización, primero de las relaciones históricas de intercambio ecológico desigual Norte-Sur y la emergencia de dinámicas similares Sur-Sur y, segundo, de la dependencia extrema del extractivismo y la explotación de materias primas que siguen caracterizando a las economías latinoamericanas. En tercer lugar, debe mencionarse el productivismo desarrollista renuente a incorporar la dimensión socio-ambiental, que caracteriza al pensamiento dominante en las elites políticas e intelectuales de América Latina, más allá del nivel discursivo en el que se suele incorporar la temática de la sustentabilidad en sus distintas vertientes, incluyendo el de la concepción del "Buen Vivir" de las tradiciones andinas. 
En relación al primer punto, la continuidad e incluso profundización de las relaciones históricas de intercambio ecológico desigual Norte-Sur y la emergencia de dinámicas similares Sur-Sur constituyen una barrera al proceso democrático de integración. Un ejemplo lo constituye la transferencia de actividades industriales "sucias", prohibidas o extremadamente reguladas en los países centrales (lo que las vuelve económica o legalmente inviables en sus territorios), hacia los países de América Latina y el Caribe. El triste ejemplo de las "papeleras" que han estado en el centro de un conflicto con ribetes casi bélicos, al menos en el plano retórico, entre Argentina y Uruguay, es un caso en cuestión. El tema de los cultivos transgénicos, cuyo cultivo y consumo se encuentra prohibido en Europa por razones eminentemente democráticas (la mayoría de la población se opone a los mismos sobre la base de la información pública acerca de los peligros potenciales de dichos cultivos) pero que en América Latina se ha expandido prácticamente sin restricciones y frecuentemente sin siquiera controles de su impacto sobre la población. Otro caso es el de la minería a cielo abierto con uso de cianuro (y otras substancias peligrosas, como el mercurio), prohibido en mayo de 2010 por el Parlamento Europeo en todo el territorio de la Unión Europea sobre la base del Principio Precautorio y de la Convención sobre la Biodiversidad adoptadas en la Declaración de Río de Janeiro de 1992 (Parlamento Europeo 2010), que sin embargo es empleado por empresas mineras, en su mayoría con matrices en países del Norte pero también de capitales regionales, en toda América Latina y en otros países del Sur Global (Sibaud 2012).

Pero los problemas de intercambio ecológico desigual también se dan entre y al interior de países en el Sur, a veces como resultado de la aplicación irregular de las normas regulatorias que favorece la "migración" de actividades contaminantes a territorios con menos control y otras veces por una reproducción de la relación entre países industrializados y países proveedores de insumos productivos entre países y regiones de América Latina y el Caribe. Un ejemplo clásico ha sido la relación entre Brasil y Paraguay en torno a la energía eléctrica generada en la represa binacional de Itaipú, una situación de desigualdad que el Presidente Lula da Silva contribuyó a revisar en negociaciones recientes con el Presidente Lugo, precisamente 
en el marco del MERCOSUR. Sin embargo, acuerdos recientes entre Brasil y varios países limítrofes para la construcción de represas, por ejemplo, están siendo observados por algunos críticos como una forma de consolidación de relaciones ecológicamente desiguales entre los países. Informes procedentes del Centro de Información del Banco, una entidad basada en Washington que se dedica a monitorear las actividades del Banco Mundial incluyendo los impactos ambientales de los proyectos financiados, alertan sobre esta dimensión importante del proceso de integración. Por ejemplo, en relación a los acuerdos entre Brasil y Perú para la construcción de represas hidroeléctricas en la amazonia peruana los críticos señalan que a Perú le correspondería el rol de proveedor de energía eléctrica y sufriría la mayor parte de los impactos ambientales en su propio territorio (Bank Information Center 2011).

En lo que concierne al segundo punto, es ampliamente reconocido que la dependencia extrema del extractivismo, incluyendo el energético (gas y petróleo) y la explotación de materias primas siguen caracterizando a las economías latinoamericanas, incluso a las más industrializadas como Brasil. Un informe reciente nos recuerda que mientras que los países industrializados, incluyendo a China, exportan principalmente manufacturas, los países "menos desarrollados" siguen dependiendo en forma extrema de la exportación de materias primas: el 70\% de los ingresos de exportación de los países latinoamericanos procede de materias primas agrícolas y minerales (PNUMA 2011: 45-46). Este es un aspecto bien conocido que no tiene sentido repetir aquí, pero vale la pena destacar algunos aspectos que son menos conocidos y que están interrelacionados con el punto anterior, el intercambio ecológico desigual. Por ejemplo, estudios recientes a nivel internacional que buscan incorporar la dimensión ambiental y, particularmente las externalidades ambientales del proceso de producción, colocan el énfasis en factores previamente ignorados en el "costo" de los productos de exportación, por ejemplo los cereales o la carne, pero también los productos industrializados. Es el caso por ejemplo del "agua virtual”, término originalmente acuñado para conceptualizar el agua incorporada en los productos agrícolas de exportación en los países del Medio Oriente y África del Norte (Allan 1998; Allan 1999; Allan 2002). El concepto de agua virtual ha disparado 
un debate muy importante a nivel internacional en el que se demuestra que la mayor parte del agua incorporada en los flujos de intercambio comercial no es contabilizada, lo cual en muchos casos toma la forma de un intercambio ecológico desigual, una transferencia de recursos no remunerada ni compensada (Chapagain y Hoekstra 2004; Chapagain y Hoekstra 2004; Hoekstra 2006; Hoekstra 2007). Lo mismo ocurre, como ya comentamos, con los flujos de contaminación incorporados en los productos comerciales (Muradian, O'Connor et al. 2002), y desde luego con las transferencias en calidad del suelo (ej. consumo de la fertilidad del suelo) que tienen lugar en estos intercambios. Como ya lo anticipara Marx a mediados del sigo diecinueve, "todo progreso de la agricultura capitalista no es sólo un progreso en el arte de esquilmar al obrero, sino a la vez en el arte de esquilmar el suelo; todo avance en el acrecentamiento de la fertilidad de éste durante un lapso dado, un avance en el agotamiento de las fuentes duraderas de esa fertilidad" (itálicas en el original; Marx 1985: 612). Países cuyas economías dependen en gran medida de las exportaciones de productos primarios como carne, cereales y otros productos agrícolas o de materias primas como minerales, maderas, y bienes similares son particularmente afectados por este tipo de intercambio ecológico desigual. Prácticamente todos los países de la región se caracterizan por este tipo de dependencia, que tiene la edad del propio capitalismo. En su fase actual, aspectos centrales del proceso de integración, como es la integración de transportes multimodal que forma parte tanto de la Iniciativa para la Integración de la Infraestructura Regional Suramericana (IIRSA) como del Proyecto Integración y Desarrollo Mesoamérica (Proyecto Mesoamérica), están profundamente comprometidos con la expansión de dicho modelo primario-exportador.

El tercer punto, íntimamente ligado con los anteriores, es el del productivismo desarrollista renuente a incorporar la dimensión socio-ambiental, que caracteriza al pensamiento dominante en las elites políticas e intelectuales de América Latina. En este punto creo que es necesario distinguir entre el plano de la urgencia inmediata y el plano de la estrategia de largo plazo que presupone el proceso de integración. En el plano más inmediato, está claro que el resurgimiento de los países latinoamericanos de la profunda crisis social, económica y política de las últimas décadas es 
un logro extremadamente significativo que debe defenderse y consolidarse. Solamente pensar en que muchos de nuestros países emergieron de la larga noche de las dictaduras para entonces hundirse en la crisis promovida por las políticas neoliberales nos pone en una situación delicada, que a muchos quizás les impide adoptar una posición crítica en relación al proceso actual de recuperación, crecimiento económico e integración regional. Sin embargo, no puede soslayarse la necesidad de la crítica a las formas dominantes de desarrollo económico, que tienden a reproducir o incluso ampliar las tendencias históricas de intercambio ecológico desigual. Una forma concreta que asume el productivismo desarrollista se manifiesta en la división del trabajo, para llamarlo de alguna manera, que suele operarse entre los ministerios de los distintos países. Una tendencia importante es que el poder, materializado entre otras cosas en poder económico, se concentre en los ministerios a cargo de actividades "productivas", como la agricultura, las industrias extractivas, o las grandes obras de infraestructura, mientras que los ministerios a cargo de temas "sociales" y "ambientales" suelen quedar rezagados, cuando no directamente subordinados en la práctica. Un ejemplo relevante es el de Marina Silva, la ex Ministra de Medio Ambiente de Brasil desde el primer mandato del Presidente Lula da Silva, quien fue recriminada incluso públicamente por Lula como responsable por la demora en el proceso de desarrollo del país. En el centro de las tensiones, que finalmente condujeron a la renuncia de la ministra en el mes de mayo de 2008, estaban la insistencia del ministerio a su cargo en la necesidad de completar el procedimiento de licenciamiento ambiental antes de autorizar emprendimientos (ej. grandes obras de infraestructura) y, fundamentalmente, la política destinada a frenar la deforestación en el Amazonas. El caso de Marina Silva es emblemático por su repercusión internacional, habiendo sido además la mano derecha de Chico Mendes y por lo tanto un símbolo no meramente de la lucha ambiental pero también de la lucha por los derechos de los trabajadores rurales y las comunidades indígenas. Sería un error reducir el caso de Marina Silva a una mera anécdota y mucho menos a una confrontación entre individuos, una ruptura entre un presidente y una ministra, o a un problema circunscrito al caso de Brasil, sino que más bien debe analizarse como parte de un proceso que asume formas diferentes a lo largo y ancho 
de América Latina y El Caribe. El ejemplo ilustra un patrón preponderante en la región, en la que por una parte gobiernos y empresas privadas asumen oficialmente el discurso de la sustentabilidad y la responsabilidad ambiental pero en la práctica la dimensión socio-ambiental continúa siendo postergada o incluso ignorada, ante lo que se percibe como cuestiones siempre más urgentes, es decir el desarrollo económico y el progreso material. Sin renunciar a la necesidad evidente de mejora material de las condiciones de vida de las sociedades latinoamericanas, es fundamental no perder la perspectiva y examinar con criterio la direccionalidad del proceso: integración (y progreso material), ¿de quiénes (quiénes son integrados)? ¿para quién se integran las regiones (quién se beneficia)? ¿a qué costo (ambiental, económico, político y socio-cultural)? ¿quién paga ese costo? ¿cómo se paga? Estas y otras cuestiones apuntan a la relación fundamental que existe entre el proceso de integración y el proceso de democratización substantiva de la región, una relación que no podemos dar por sentada.

\section{La injusticia y la desigualdad ambientales}

Proposición $\mathrm{N}^{\circ}$ 4. La destrucción de la base material que acompaña el proceso de intercambio ecológico desigual se presenta como una doble amenaza para el proceso de integración democrática en la región. Por una parte, la destrucción de la base material por definición constituye un obstáculo al propio modelo de integración fundado en la profundización de relaciones capitalistas, ya que erosiona los mismos fundamentos de la producción. Por otra parte, el proceso se ha constituido en uno de los motores de la producción y reproducción de desigualdades estructurales, en la forma de injusticia y desigualdad socio-ambientales, y como tal, en un obstáculo crucial al proceso de profundización de la democracia substantiva.

Como se comentó anteriormente, la noción de "límites naturales" a la producción, es decir la toma de conciencia de que a pesar de la enorme capacidad científica y tecnológica desarrollada (y potencialmente alcanzable) 
por los seres humanos existen fronteras que impone la base material de las sociedades, no es meramente el producto de ambientalistas utópicos y profetas del apocalipsis ecológico sino que actores ilustrados, para llamarlos de alguna forma, entre los propios defensores de la democracia capitalista coinciden en el análisis, como en el caso ya mencionado del Club de Roma. En el mismo sentido, un informe reciente del Panel Internacional de Recursos de Programa de las Naciones Unidas para el Medio Ambiente (PNUMA) indica lo siguiente:

"al igual que en informes previos del Panel, la sobrexplotación de recursos, el cambio climático, los cambios en el uso del suelo, y la pérdida de biodiversidad se han elevado a la cima de las principales preocupaciones internacionales. Un resultado es que 'la sustentabilidad' se ha convertido en un imperativo social y económico entre los gobiernos, las organizaciones internacionales y las empresas. Líderes en estos sectores ahora comprenden que el progreso hacia una economía más sustentable requiere una reducción absoluta en el uso de recursos a nivel global, mientras que el bienestar de los seres humanos demanda simultáneamente una expansión de las actividades económicas y una disminución de los impactos ambientales. El dilema de expandir las actividades económicas y simultáneamente reducir la tasa de uso de recursos y el impacto ambiental que tales usos puedan tener presenta un desafío muy serio a la sociedad" (PNUMA 2011: 7-8).

En relación a nuestro tema, inmediatamente surgen algunas cuestiones relacionadas con las conclusiones de este informe. ¿Es posible plantearse esta noción de "límites" en el contexto de la integración regional de América Latina y el Caribe? ¿No sería la aceptación de la existencia de tales límites una tácita aceptación de la imposibilidad del desarrollo, es decir, de la "expansión de las actividades económicas" que el propio informe del PNUMA reconoce como un requisito para garantizar "el bienestar de los seres humanos" en nuestra región? En este sentido, un tema preocupante a plantearse es en qué medida los "líderes" políticos y empresariales, para usar el lenguaje del informe del PNUMA, del proceso de integración latinoamericano 
y caribeño también comprenden la necesidad de confrontar el dilema que presenta la necesidad de garantizar el bienestar de la población y simultáneamente proteger la base material de las sociedades de la región. El hecho de que una parte significativa de los conflictos sociales que tienen lugar en toda la región estén precisamente conectados en una forma u otra con la percepción y/o realidad efectiva de crecientes injusticias y desigualdades ambientales causadas entre otros factores por la minería a cielo abierto, las fumigaciones de cultivos transgénicos, la deforestación incontrolable, la biopiratería a veces negociada o por lo menos tolerada por los gobiernos, la imposición frecuentemente inconsulta e inclusive forzosa de la construcción de obras masivas de infraestructura que frecuentemente desplazan poblaciones enteras destruyendo sus lugares ancestrales y transformando irreversibles los ecosistemas, o la continua contaminación del aire, el suelo y el agua por actividades económicas no controladas o pobremente reguladas, sugiere que este dilema asume dimensiones aún más colosales en el contexto de nuestra región (Alimonda 2002; Jasanoff 2006; Brailovsky y Foguelman 2007; Kuecker 2007; van Dijck y den Haak 2007; Carruthers 2008; Newell 2009; Urkidi Azkarraga 2010; Zhouri y Laschefski 2010; Pieck 2011). Inclusive si asumimos que existen niveles de conciencia crecientes en los segmentos de liderazgo político y empresarial de América Latina y el Caribe sobre la naturaleza del desafío que se confronta, la evidencia sugiere que entre la toma de conciencia del dilema y la capacidad y/o voluntad de implementar acciones concretas al respecto existe un enorme vacío. Vaya como pequeño, aunque no por eso menos impresionante, ejemplo el caso del fallido intento de pasar una ley de protección de bosques en la provincia de Córdoba, Argentina en el año 2010. Se estima que Córdoba perdió en el curso del siglo veinte el 95 por ciento de su bosque serrano nativo, y el objetivo central de la ley propuesta por una alianza de sectores provinciales era impedir el avance de la deforestación promovido por la incursión acelerada de la agricultura sojera y otras actividades económicas (Barri y de Luca 2009). La ley para proteger ese 5 por ciento de bosque nativo sobreviviente no logró ser aprobada y en su lugar la legislatura provincial pasó otra ley que ha sido denunciada por una amplia gama de actores pues convalida la continua deforestación de la provincia, a punto tal que la propia Defensoría 
del Pueblo de la Nación acaba de expresarse al respecto (Redacción La Voz del Interior 2012). Desde ya, no se trata meramente de una confrontación entre ambientalistas preocupados con la preservación de la naturaleza en abstracto, por un lado, y de gobiernos y empresarios creadores de riqueza por el otro, como a veces se suelen plantear estas controversias en nuestras sociedades. Córdoba, una provincia semidesértica, ha visto seriamente agravada la situación ambiental en las últimas décadas y confronta entre otras cuestiones una crisis hídrica de gran magnitud, relacionada en gran medida con la deforestación extrema y el avance incontrolado de la agricultura intensiva. No solo está en juego la sustentabilidad del propio modelo productivo cordobés sino que la misma calidad de vida de las personas ya está siendo seriamente afectada lo cual sigue dinamizando recurrentes conflictos contra la injusticia y la desigualdad socio-ambientales derivadas de estos procesos. El ejemplo de Córdoba es un caso prominente, pero de ninguna manera constituye una excepción o rareza y más bien ejemplifica un patrón preocupante a nivel regional, ya que los conflictos socio-ambientales han pasado a ocupar un lugar central en nuestras sociedades y tienden a expresar la existencia de profundas contradicciones en el proceso de democratización regional.

\section{Conclusiones: democracia substantiva y lucha socio-ambiental}

En función de lo anterior cabe plantearse algunas cuestiones adicionales: ¿en qué medida el proceso de integración en marcha presupone la profundización de los procesos de injusticia y desigualdad socio-ambiental? ¿Es posible plantearse un modelo de integración democrático que tenga como eje central el abatimiento de la injusticia y la desigualdad simultáneamente en las dimensiones social y ambiental? Por momentos, la dirección que toma el proceso en algunos países de la región, donde la movilización de comunidades afectadas por la minería, las represas, las fumigaciones, y otros problemas similares ha sido respondida incluso con represiones violentas en los casos más extremos y con discursos de desaprobación (por ejemplo, "quienes se oponen a la minería, se oponen al progreso"), nos lleva 
a plantearnos una serie de cuestiones potencialmente importantes para el futuro de la integración regional.

¿Será que en América Latina y el Caribe implícitamente se acepta la hipótesis del politólogo norteamericano Ronald Inglehart según la cual la preocupación por cuestiones ambientales pertenecería a la etapa pos-material del desarrollo social, una característica avanzada que aún se encuentra en el futuro distante para la mayoría de las sociedades menos desarrolladas (Inglehart 1971)? Aunque la hipótesis de Inglehart ha sido consistentemente rechazada en el plano del conocimiento (Brechin y Kempton 1994; Brechin y Kempton 1997; Dunlap y Mertig 1997; Martinez-Alier 2002), la práctica política, incluyendo la de los gobiernos progresistas en relación a los ejemplos mencionados más arriba, frecuentemente parece asumir dicha hipótesis como presupuesto de su política ambiental y de desarrollo.

En relación con lo anterior, ¿será posible reconciliar la justicia y la igualdad sociales con la justicia y la igualdad ambientales? ¿Cuál es el "límite" o "umbral" aceptable de tolerancia a la injusticia y la desigualdad ambientales cuando estas parecen incompatibles con la justicia y la igualdad sociales? ¿En qué situaciones sería legítimo subordinar la justicia y la igualdad ambientales a la justicia y la igualdad sociales? ¿Es posible plantearse esta disyuntiva desde una posición de democracia substantiva, no meramente formal? Estas preguntas revisten gran relevancia, lo que queda de manifiesto particularmente en las grandes disyuntivas éticas y políticas que confrontan los gobiernos progresistas de la región en relación a las contradicciones entre sus políticas ambientales y de desarrollo económico. Valgan como ejemplos los conflictos generados por el proyecto de transvase del Río San Francisco en Brasil, el gasoducto trans-amazónico, la construcción de represas y la minería a cielo abierto en los diversos países de la región, la introducción de cultivos transgénicos, la continua deforestación, la destrucción de la biodiversidad y de la fertilidad de las mejores tierras del continente, la privatización y mercantilización del agua y sus servicios esenciales (aunque con otros nombres, como las "asociaciones público-privadas"), la gestión pública de los desastres "naturales", el tema nuclear (no 
debatido seriamente por los países de la región), o el impacto social de los procesos de cambio climático.

Con el afán de contribuir al debate crítico, en primer lugar, sería conveniente examinar la existencia de obstáculos epistemológicos e inobservables que frenan tanto el avance de la producción de conocimiento como la acción práctica sobre estos problemas. Específicamente es importante revisar los presupuestos de las tradiciones desarrollistas que predominan en la región, de gran vigencia en la actualidad, a la luz de los debates recientes a nivel internacional, incluyendo el debate latinoamericano, sobre la insustentabilidad del modelo de desarrollo prevaleciente. En segundo lugar, es importante discutir el repliegue del pensamiento y la acción políticos al "presente", con un predominio de las visiones hodiocéntricas, ahistóricas, características de la economía neoclásica y de las tecno-ciencias pero que en las últimas décadas han permeado también a las ciencias sociales y a las estrategias de los sectores progresistas comprometidos con la justicia social. ¿Cómo recuperar la capacidad de distanciamiento crítico con respecto a la realidad inmediata, la perspectiva histórica, el análisis de los futuros posibles? En tercer lugar, y para concluir, cabe plantearse el problema en su dimensión ética, y muy especialmente en relación al proceso de autonomización del plano moral y la subordinación de los valores al plano instrumental en nombre del "realismo", la "eficacia" y el pragmatismo político que caracterizan en gran medida la relación entre política ambiental y desarrollo social tanto en la teoría como en la práctica. Una política democrática que tienda a abarcar el conjunto, en perspectiva de largo plazo, requiere la superación de las contradicciones aparentemente irresolubles entre los requerimientos de la justicia y la igualdad ambientales y sociales. A su vez, en el plano intelectual este desafío requiere una ruptura epistemológica que permita alcanzar niveles más elevados de coordinación interdisciplinaria en la producción de conocimiento científico sobre las relaciones sociedad-naturaleza, un proceso en el que las ciencias sociales latinoamericanas están llamadas a jugar un papel central. 


\section{Referencias}

Alimonda, H., Ed. (2002). Ecología Política. Naturaleza, Sociedad y Utopía. Buenos Aires, Consejo Latinoamericano de Ciencias Sociales, CLACSO.

Allan, J. A. (1998). "Watersheds and problemsheds: Explaining the absence of armed conflict over water in the Middle East." Middle East Review of International Affairs 2(1): 49-51.

Allan, J. A. (1999) Israel and water in the framework of the Arab-Israeli conflict.

Allan, J. A. (2002). The Middle East Water Question. Hydropolitics and the Global Economy. London and New York, Tauris.

Bank Information Center (2011). "Represa Hidroeléctrica Inambari." Disponible en: http://www.bicusa.org/es/Project.10078.aspx.

Barri, F. y N. de Luca (2009). El valor de los servicios ambientales. La Voz del Interior, 15 de mayo de 2009. Córdoba: A8.

Brailovsky, A. E. y D. Foguelman (2007). Memoria Verde. Historia Ecológica de la Argentina. Buenos Aires, Editorial Sudamericana.

Brechin, S. R. y W. Kempton (1994). "Global environmentalism: a challenge to the postmaterialism thesis?" Social Science Quarterly 75(2): 245-269.

Brechin, S. R. y W. Kempton (1997). "Beyond postmaterialist values: national versus individual explanations of global environmentalism." Social Science Quarterly 78(1): 16-20.

Bunker, S. G. (1984). "Modes of extraction, unequal exchange, and the progressive underdevelopment of an extreme periphery: the Brazilian Amazon, 1600-1980." American Journal of Sociology 89(5): 1017-1064. 
Carruthers, D. V., Ed. (2008). Environmental Justice in Latin America. Problems, Promise and Practice. Cambridge, MA, and London, UK, MIT Press.

Chapagain, A. K. y A. Y. Hoekstra (2004). Water Footprints of Nations. The Value of Water Research Report Series. Delft, UNESCO.

Chapagain, A. K. and A. Y. Hoekstra (2004). Water Footprints of Nations. Appendices. The Value of Water Research Report Series. Delft, UNESCO.

Club de Roma (2012). “2012: 40 years Limits to Growth. What Was the Real Message of Limits to Growth?”. Disponible en: http://www.clubofrome. org/.

Cornia, G. A. (2010). "Income Distribution under Latin America's New Left Regimes.” Journal of Human Development and Capabilities 11(1): 85-114.

Crosby, A. W. (1988). Imperialismo ecológico. La expansión biológica de Europa, 900-1900. Barcelona, Editorial Crítica.

De Vries, B. y J. Goudsblom (2002). Mappae Mundi. Humans and their Habitats in a Long-Term Socio-Ecological Perspective. Myths, Maps, and Models. Amsterdam, Amsterdam University Press.

Delgado Wise, R. D. y M. Ortega Breña (2006). "Migration and Imperialism: The Mexican Workforce in the Context of NAFTA." Latin American Perspectives 33(2): 33-45.

Dunlap, R. E. y A. G. Mertig (1997). “Global environmental concern: an anomaly for postmaterialism." Social Science Quarterly 78(1): 24-29.

CEPAL (2009). Social Panorama of Latin America 2008. Santiago de Chile, Comisión Económica Para América Latina y el Caribe. 
Elias, N. (1978). What is Sociology? Londres, Hutchinson.

Parlamento Europeo (2010). European Parliament resolution of 5 May 2010 on a general ban on the use of cyanide mining technologies in the European Union. Luxemburgo, Parlamento Europeo.

Falcon, W. P. y C. Fowler (2002). "Carving up the commons-emergence of a new international regime for germplasm development and transfer." Food Policy 27(3): 197-222.

Farah, I. y L. Vasapollo, Eds. (2011). Vivir Bien: ¿Paradigma no Capitalista? La Paz, Bolivia, Postgrado en Ciencias del Desarrollo de la Universidad Mayor de San Andrés (CIDES-UMSA) y Departamento de Economía de la Universidad de Roma "La Sapienza".

Fischer-Kowalski, M. y H. Haberl, Eds. (2007). Socioecological Transitions and Global Change. Trajectories of Social Metabolism and Land Use. Cheltenham, Gloucestershire, UK, Edward Elgar Publishing.

Gammage, S. (2006). "Exporting People and Recruiting Remittances: A Development Strategy for El Salvador?" Latin American Perspectives 33(6): 75-100.

Gosman, E. (2009). Furia en Brasil: llegan residuos tóxicos clandestinos de Inglaterra. Clarín. Buenos Aires, Grupo Clarín.

Gudynas, E. (2011). "Buen Vivir: Germinando alternativas al desarrollo." América Latina en Movimiento(462): 1-20.

Guha, R. y J. Martínez-Alier (1997). Varieties of Environmentalism. Essays North and South. Londres, Earthscan.

Helfrich, S., Ed. (2002). La Vida en Venta. El Salvador, Fundación Heinrich Böll Editions. 
Hoekstra, A. Y. (2006). The Global Dimension of Water Governance: Nine Reasons for Global Arrangements in order to Cope with Local Water Problems, The Value of Water Research Report Series. Delft, UNESCO.

Hoekstra, A. Y. (2007). Human Appropriation of Natural Capital: Comparing Ecological Footprint and Water Footprint Analysis. The Value of Water Research Report Series. Delft, UNESCO.

Hornborg, A. (1998). "Towards an ecological theory of unequal exchange: articulating world system theory and ecological economics." Ecological Economics 25: 127-136.

Inglehart, R. (1971). "The Silent Revolution in Europe: Intergenerational Change in Post-Industrial Societies." The American Political Science Review 65(4): 991-1017.

Jasanoff, S. (2006). "Biotechnology and Empire: The Global Power of Seeds and Science." Osiris 21(1): 273-292.

Jorgenson, A. K. y B. Clark (2009). "The Economy, Military, and Ecologically Unequal Exchange Relationships in Comparative Perspective: A Panel Study of the Ecological Footprints of Nations, 1975-2000." Social Problems 56(4): 621-646.

Kuecker, G. D. (2007). "Fighting for the Forests: Grassroots Resistance to Mining in Northern Ecuador." Latin American Perspectives 34(2): 94-107.

López-Calva, L. F. y N. C. Lustig, Eds. (2010). Declining Inequality in Latin America. A Decade of Progress? Nueva York, Brookings Institution Press y PNUD.

Martinez-Alier, J. (2002). The Environmentalism of the Poor. A Study of Ecological Conflicts and Valuation. Cheltelham, UK, and Northampton, Ma, USA, Edward Elgar. 
Martínez-Alier, J. y A. Olivares (2003). ¿Quién debe a quién? Deuda externa y deuda ecológica. Barcelona, Editorial Icaria.

Martins, H. (1996). Hegel, Texas e outros Ensaios de Teoria Social. Lisbon, Século XXI.

Martins, H. (1998). 0 deus dos artefatos: sua vida, sua morte. Tecnociência e Cultura. Ensaios sobre o Tempo Presente H. Reis de Araújo. Sao Paulo, Estação Liberdade: 149-168.

Martins, H. (1998). "Risco, incerteza e escatologia. Reflexões sobre o experimentum mundi tecnológico em curso (I).” Episteme 1(1): 99-121.

Martins, H. (1998). "Risco, incerteza e escatologia. Reflexões sobre o experimentum mundi tecnológico em curso (II).” Episteme 1(2): 41-75.

Marx, K. (1985). El Capital. Crítica de la Economía Política. México DF, Siglo XXI.

McNeill, J. (2000). Something new under the Sun. An Environmental History of the Twentieth Century. Londres, Penguin.

Meadows, D. H., D. L. Meadows, J. Randers, y W. W. Behrens III (1972). The Limits to Growth. Washington, D.C., Potomac Associates, New American Library.

Muradian, R., M. O'Connor, y J. Martínez-Alier (2002). “Embodied Pollution in Trade: Estimating the environmental load displacement of Industrialized Countries." Ecological Economics 41(1): 51-67.

Nelson, G. C. y R. D. Robertson (2008). "Green Gold or Green Wash: Environmental Consequences of Biofuels in the Developing World." Review of Agricultural Economics 30(3): 517-529. 
Newell, P. (2009). "Bio-Hegemony: The Political Economy of Agricultural Biotechnology in Argentina." Journal of Latin American Studies 41(1): 27-57.

O'Connor, J. (1998). Natural Causes. Essays in Ecological Marxism. New York, Guilford Press.

OCDE (2010). Tackling Inequalities in Brazil, China, India and South Africa. Paris, OCDE Publishing.

Peritore, N. P. and L. B. Niles (1992). "El surgimiento del cartel biotecnológico.” Revista Mexicana de Sociología 54(2): 101-131.

Pieck, S. K. (2011). "Beyond postdevelopment: civic responses to regional integration in the Amazon." Journal of Cultural Geography 28(1): 179-202.

Redacción La Voz del Interior (2012). La Defensoría le pide a Córdoba que cambie su ley de bosques. La Voz del Interior, 14 de enero de 2012. Córdoba.

Rice, J. (2007). "Ecological Unequal Exchange: Consumption, Equity, and Unsustainable Structural Relationships within the Global Economy." International Journal of Comparative Sociology 48(1): 43-72.

Rice, J. (2007). "Ecological Unequal Exchange: International Trade and Uneven Utilization of Environmental Space in the World System." Social Forces 85(3): 1369-1392.

Rice, J. (2009). "North-South Relations and the Ecological Debt: Asserting a Counter-Hegemonic Discourse." Critical Sociology 35(2).

Roberts, J. T. y B. C. Parks (2007). "Fueling Injustice: Globalization, Ecologically Unequal Exchange and Climate Change." Globalizations 4(2): 193-210. 
Schnaiberg, A. (1980). The Environment: From Surplus to Scarcity. New York, Oxford University Press.

Schnaiberg, A. y K. A. Gould (1994). Environment and Society: The Enduring Conflict. New York, St. Martin's Press.

Shiva, V. (2001). Biopiratería. El saqueo de la naturaleza y el conocimiento. Barcelona, Editorial Icaria.

Sibaud, P. (2012). Opening Pandora's Box. The New Wave of Land Grabbing by the Extracting Industries and the Devastating Impact on Earth. London, The Gaia Foundation: 56.

Simms, A. (2009). Ecological Debt: Global Warming and the Wealth of Nations. London, Pluto Press.

Suárez Salazar, L. y M. Ortega Breña (2007). “The New Pan-American Order: The Crisis and Reconstitution of the U.S. System of Global Domination." Latin American Perspectives 34(1): 102-111.

Taylor, J. (1994). “The challenge of sustainable development." Regulation 17(1): 35-50.

PNUMA. (2011). Decoupling natural resource use and environmental impacts from economic growth, A Report of the Working Group on Decoupling to the International Resource Panel. Paris, PNUMA.

Urkidi Azkarraga, L. (2010). Environmental Justice and the Politics of Scale in Latin American Social Movements against Gold Mining. The Cases of Pascua-Lama in Chile and Marlin in Guatemala. Instituto de Ciencia y Tecnología Ambientales. Barcelona, Universidad Autónoma de Barcelona. Doctorado en Ciencias Ambientales. 
van Dijck, P. y S. den Haak (2007). Construcción Problemática. IIRSA y las Asociaciones Público-Privadas en la Infraestructura Vial. Amsterdam, Centro de Estudios y Documentación Latinoamericanos (CEDLA).

Vecchio, R. (2007). Bioprospecting: Who has rights to nature's cures? The Seattle Times, 12 de enero de 2007, Seattle.

Zhouri, A. y K. Laschefski, Eds. (2010). Desenvolvimento e Conflitos Ambientais. Belo Horizonte, Editora UFMG. 


\section{José Esteban Castro}

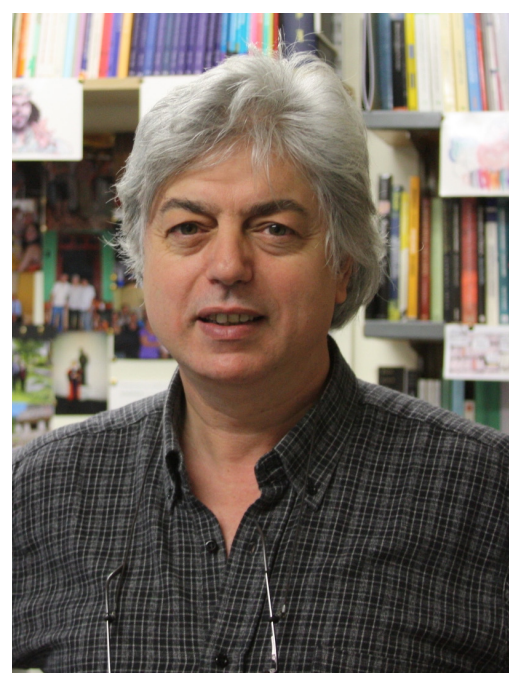

Catedrático (Full Professor) em Sociologia na Universidade de Newcastle, Reino Unido, desde 2005. Previamente foi Pesquisador Principal Associado na Escola de Geografia e Meio Ambiente e no Centro de Estudos Brasileiros da Universidade de Oxford (2001-2004), e Professor em Estudos sobre o Desenvolvimento na London School of Economics and Political Science (2000-2001). Castro tem uma formação interdisciplinar nas ciências sociais: é Licenciado e Professor em Sociologia pela Universidade de Buenos Aires (19831988), estudou Psicologia na mesma Universidade (1984-1990), obteve um Mestrado em Ciências Sociais pela Faculdade Latino-americana de Ciências Sociais (FLACSO) Sede México (1990-1992) e é Doutor em Ciência Política pela Universidade de Oxford (1993-1998). Ele é Membro Correspondente da Academia Mexicana de Ciências. Castro tem realizado tarefas de pesquisa e docência em diversos países, incluindo Argentina, Brasil, Espanha, Colômbia, México, o Reino Unido, e Portugal. Coordena a Rede Internacional WATERLAT-GOBACIT (http://www.waterlat.org), dedicada à pesquisa, a docência e a ação prática em relação à política e a gestão da água e seus serviços. Ele é professor visitante da Universidade Estadual da Paraíba (UEPB) desde 2008. 